\title{
Analysis of the Influence of Primitive Art on Contemporary Art
}

\author{
Ge Cheng ${ }^{1}$, Lu Cheng ${ }^{2}$ \\ ${ }^{1}$ Shanghai Sibo vocational and Technical college, China \\ ${ }^{2}$ ShangHai Publishing and Printing College, China
}

Keywords: primitive art, abstraction, simplification, painting, sculpture, design, symbolization.

\begin{abstract}
The original art simple naive is a source of inspiration and the origin of art, even the future of art exploration breakthrough window, characteristics of the original art, looking for their unique connotation, is a source of the development of modern art, but also the modern, A source of design inspiration, echoing the return of contemporary art or primitive art use, and repetition is not a simple simulation, but a process of art spiral, is a continuation of the original art and development, is the heritage context, it is also the starting point of the future artistic breakthrough.
\end{abstract}

\section{Introduction of Primitve Art}

Primitive art refers to the total amount of art created by human beings in prehistoric society, which is related to life, survival and religion. It is the crystallization of human beings' material creation and spiritual belief in primitive society. People in primitive society, in order to adapt to the nature of survival, through the practice of Labor Transformation Tools continuously, create different types and various patterns such as pottery and bone, because of the fear of nature by the totem religion related, tomb murals painted pottery, these primitive art has a unique geographical and ethnic origin the influence of culture and art active later far, generally speaking, the original art is mainly the primitive man's original logical thinking and imagery thinking, through a simple intention of mystery or hope things express direct description, this description is simple and clumsy imagination, No smelting logic thought judgment reasoning process, so the artistic performance is directly simple intention, but also can be said to be the rudiment of cognition, form of performance art, the expression of embryonic origin, like the young children of the world and the fuzzy understanding of children's painting naive random combination of expression. With the development of social times, the thinking of the original art is gradually abstracted and reasoned by the thinking of the civilized age, and the concepts are transferred and replaced. The primitive art is gradually becoming figurative and rich and symbolic. To the modern experience after the industrial revolution happened to turn the world upside down changes in art, different cultural art, art trend of reform in different, different ideological and cultural impact, positive mood art abstract deformation technique and so on graffiti began to explore and develop new artistic expression, some even inadvertently in the trend of primitive art intends to return,At different time in the form of test and find new ways to break through, so the original art simply childish is a source of origin and Inspiration of art culture, even the future of art exploration breakthrough window, characteristics of the original art for their unique connotation, is a symbol of our today with the development of modern art the source is a source of inspiration for modern design, is the heritage context, it is of the future artistic breakthrough point.

\section{The influence of primitive art on Contemporary Painting}

Influence of original art on contemporary art, the ancestor of the Chinese nation in the primitive society also created the original art with its unique features, the most striking is the art category of Yangshao culture pottery and sculpture, as $5500 \mathrm{BC}$ to $3000 \mathrm{BC}$, it is the raw material of fine greasy sticky loess, the paint is painted natural ochre, red or mineral pigment, making high temperature of 1000 degrees in the kiln, the kiln burning chamber seal is not tight, iron oxide pottery in the soil after the formation of the orange, red or brown red color pottery, the sculpture was dark and red. Banpo 
types of pottery mostly round or flat bottom of the pot or round lip drum belly tank more, style simple and heavy, The decoration is human face, fish, deer, geometric patterns are parallel lines, parallel diagonal form two sides successive pattern with triangular face, good decorative effect, human face fish is the most representative pattern of man's face is round, the lines represent eyes, with triangular nose said the forehead, chin, is the ancient people of the simple description, and then in the head and ears around the painted triangle, each triangle three side depicts a short thin edge, or directly painted fish in the head or ear on both sides of the fish body, simple, entire, weird patterns the mysterious and combination of grotesque, combination of thinking itself patterns of origin has been mocongkaozheng, perhaps is a positive portrayal of the fish, the fish head is the human face, perhaps to harvest fish hanging on the combination head to celebrate, May the Banpo people and ancient totem worship and the life, as a kind of celebration or fishing or fish possessed religious makeup image, This simple and direct in the pelvic floor behave like children on any combination of world children's paintings, it contains the meaning of today is not the way we thought is complex, to harvest different combination of painting fishing together, and the relationship between the performance of fish instead of other elements, combination of abstraction and simplification, the formation of a skill performance space stacked, express a sense only to the meaning of the mysterious properties can only be sensed, but appears to become a historical symbol of a proprietary. To Majiayao pottery period, have not been satisfied with the painted pottery art of shape change itself, this period pottery decoration gradually increased, and the development of pottery decorative painting, mainly black color with red color, and some even adorned the device, texture smooth curve of vivid, complex distribution of dense but are divided into primary and secondary, with strong decorative effect, symbolic meaning and spirit reflects the spirit of the pursuit of higher decorative expression more primitive world. For example, the inner wall of dance basin - basin along the mouth of a circle is a primitive array dance chart, each group of five people, holding each other hands in a row, behind a bun, waist oblique lower point has a similar clothes with animal tail streamers, on both sides of the outer arm are drawn into the line, said swing feeling fluctuation by the people, therefore in the formation of online Kuang, circle two party continuously, with the original flavor and rich sense of decoration, the body is very simplified, almost symbolic, is widely used in the decorative techniques of folk art, has a great influence on modern art.

As the most prominent original cave paintings, Alta Mira of northern Spain and southwestern France cave paintings the Lascaux Cave murals, depicting various walking running animal lines in the cave wall, focus on the object with the typical characteristics of external shape of the grasp, abandoned the details of the characterization, dynamic generalization ability is very strong, simple lines the naive approach is realistic, vivid, showing a clever clumsy in. People often simply linear "composition", more positive, more animal silhouette. The upper limb movements such as high diversity character extension, or throwing, or waving more primitive art out of human artistic creation instinct and sensitive, contains a rough ignorance of life consciousness, the painting is in order to survive or religious and practical, to identify animal and animal to hunt. Primitive rock evolution is realistic to symbolic realistic style from the image to simple to abstract symbols, the picture looks strong and bold, tend to simplify, Zhizhuo, extensive, like a modern abstract painting.

Although the original art and modern art of painting has thousands of years of distance in the understanding of the world, values and aesthetics, but in some performance have different approaches but equally satisfactory results counseled this is because on the one hand, the development of modern painting is no longer a reproduction of emotional things, many western artists began to diversify from the traditional thought of expressing feelings the shackles of realism and art ways of the complex, the exploration of modern art genres of painting language and form, and with the combination of philosophy, all kinds of painting language expression Abstract simplified symbolic, and combination of strange, such as Daly's painting line simplified abstract symbolic on the basis of performance, more close to an irrational, illogical crazy dreams, will be distorted by the objective world, Fine photo to express a kind of crazy reality, his work has inspired people's imagination to explore the subconscious, graffiti painting language symbols similar to the original art, and primitive art has aroused the agree without prior without previous consultation, seeking inspiration to the original 
painting art trend.

On the other hand, the modern art of traditional performance, the formation of a new painting style and genre, from the original painting art skills, modern art masters Matisse said: "I seek, is the most important performance... I can't tell the difference between my feelings for life and the way I express my feelings. "Matisse from the original pursuit of dynamic, form a strong color, a powerful and unconstrained style point of view, gradually developed into the pursuit of simple simple, pure and quiet, decorative style tends to the original state, the pursuit of a pure original childish and natural expression, the original natural art affects the development of modernism. In the exploration of art, Picasso turned to borrow the form of primitive art to get inspiration and inspiration, which was a typical case,In 1906 Picasso was affected by the black, bold, original sculpture form strong, to stimulate and shock to his powerful, research and use this art form in the 1907 "by strong girls" is the creation of paintings become three-dimensional style of milepost works. Picasso's Cubist painting style, from the original art for inspiration to rational and abstract objects, re formation, combination, bring people a newer and more profound feelings, the creation of epoch-making works and achieved great success at the same time, to promote the language of painting more forms and paintings the performance of force.

\section{Influence of primitive art to contemporary sculpture art,}

Banpo pottery pattern is another fish, a fish tank in the drum abdomen painted, fish mouth wide, a black fish is small, with a sketch of the various straight and slightly curved curve, some fish fins filled intermediate and triangular pigment, with contrast the surface and line, this fish is of realistic life, is the primitive people to fish description and modification, the whole design is simple and rich, there are positive and negative strength contrast straight and curve of yin and Yang, the whole pattern is simple and primitive and mysterious. In the Tang and Song Dynasties, painters painted poultry were painted and painted entities, Contemporary painting has been gradually abandoned since the Ming and Qing Dynasties on the object's detailed description, but the concise lines, the combination of the abstract painting, to express a concept and idea, this method can be inspired by this plexus fish patterns, modern design has also been a lot of inspiration and inspiration, the similar impressionistic performance and the pattern of primitive society in the contrast and agree without prior without previous consultation, straight and curved of yin and Yang in our contemporary art and design is widely used in.The last round, relief is an entity, is bulky and Reiki enough, with the continuous development of modern art, the art of abstract performance has become a mainstream form of sculpture, also show the plexus gradually transformed into the combination itself the main entity structure, then the variable structure for art processing lines, leaving a lot of empty space and the art of the combination of lines, in contrast to the actual merits of yin and Yang, let people through the void can extend the infinite imagination space, and integration with the surrounding environment, the most famous example is Henry Moore sculpture, concise and abstract form of his sculpture works are full of expressive force, also by contact and twist connection, separation, promotion, integration in various ways such as using a combination to show a motionless statue is full of mysterious tension and vitality,Exudes a unique language connotation, Moore in studying the carving inclined holes form, make the space through sculpture, a part of the space as sculpture entities, but also as the outer space in the virtual crossing, the mysterious strength and surrealist overtones, and this is the traditional Chinese the actual situation and the pros and cons of Art advocates the theory of yin and Yang is the same as yours, from the plane point of view, the human body painting reduced to fragments of Moore sculpture and Picasso is clever Tiancheng different approaches but equally satisfactory results. "The arch" this piece is one of his representative works, which is a static, strong, energetic, have the power to contain bloom as their lives, the sculpture from a bone formation after inspiration, seem to see the primitive society of the Stonehenge, he said that his work is to learn from nature, artists we should learn from the original art. These great works, compared with the original art at the end, have the same taste, but also produced a new era of unique new connotations,Let art get more road development. Brown Cousy is a Romania born French sculptor, also influenced by early cubism and primitive African sculpture, made of different materials to simplify the modeling of the 
sculpture, masterpiece "the kiss", "endless" column, his sculpture design line is very simple, but there is a quiet mood and folk art features in the west, occupies a unique position in modern sculpture.

\section{Influence of primitive art to contemporary art design}

The jade stone is composed of primitive society to the development process, the grinding stone, craftsmen took some transparent color and light and beautiful stone products wear elaborate ritual. These products shape symmetry, compact structure, light green color, or pan green or yellowish tendency the common form of Bi, Huang Yuan, Yue Zhuhuan tube, ritual and semi cylindrical trapezoidal animal shape, Yu Zong, Yuhuan, bracelets and so on shape more rigorous, wall smooth, neat edge trace, some surface is decorated with exquisite patterns, it is as the acme of perfection Art beats nature.(jade Zong shaped facade is divided into several sections with roundness inside but squareness outside, and only a short body section, each section of column four corner to corner the edge to the center line, symmetrical deformation carved animal faces, Yuzong center diameter shot dig material skilled, large jade chongkal more than $30 \mathrm{~cm}$ aperture, most of only $12 \mathrm{~cm}$ aperture edge edge warping, the hole wall is smooth, from the front, it is a middle vertical slot, and transverse grooves to jade were divided into four blocks, each block has two characterizations of a small concentric circle, the outside is not concentric with a large oval connection a small piece of trapezoidal projection, and the turning point is symmetrically connected from the front lines, these combinations like stars in the universe mysterious position description,With a sense of time and space, but just from the side is a three-dimensional terrorist animal faces, this may be the later summer bronze Taotie like shape, the shape and texture is full of mysterious and dignified religious atmosphere, these jade exquisite carving skills, pattern composition complex, the deep meaning of the modern art design profound influence.

Contemporary society is rich in material, people pursue spiritual, religious and aesthetic feelings and pursue cultural works of art, highlighting personal taste and aesthetic style. Some exquisite handicraft of modern design also uses the original art craft shape and decoration, many boutique design such as contemporary glass technology is from the original arts and crafts from learned many elements, the original pattern engraved on a simple color transparent glass, which has a strong flavor of primitive totem and a new era. Style, full of the characteristics of the new era to primitive culture and ancient glaze. There are modern pottery shape and ornamentation, abandoning the traditional dull obscure ornamentation, the original shape and modern relaxed and graceful pattern together, resulting in a new style, in addition to the original art style of interior design is graphic design and so on art has profound influence, increase the art culture heritage the essence of the culture of ancient art, let the soul renewed vitality in new era.

\section{Conclusion}

Throughout the development of the art history, there will be a pioneer in the trend of exploration and fishing back in the history of the scattered ancient art essence, inheritance develop and find new elements to draw nourishment from them, for the development of art of the era seeking a breakthrough, it is the pursuit of the essence of human emotion feedback, but also continue to pursue the highest human practice the realm of beauty, contemporary art again falling in the wild times of the original charm and mystery to discover and show their deep human subconscious spirit and aesthetic feeling, is the return of contemporary art or original art uses the echo and repetition is not a simple simulation, but a process of art spiral, is a continuation of original art and development, primitive art is the human baby period to the world the beauty of modern art, it is also to show the inner goodness Beauty.

\section{Reference}

[1] JJade culture in the Neolithic Age -- Liangzhu culture http://www.luxee.com/htmlNews/2005/7/ 12/1003306.html 